and will never develop overt disease. Understanding differences in immune cell physiology between ANA+healthy individuals and individuals with clinical SLE remains a critical goal in the understanding of SLE pathogenesis across ethnicities.

Methods Blood specimens and information on disease activity were collected from European (EA) and African American (AA) individuals classified and matched in groups as ANAhealthy controls $(n=24)$, ANA thealthy $(n=24)$ or SLE patients $(n=24)$. Single-cell analysis of cell surface markers was completed by mass cytometry on PBMCs and cellular heterogeneity was visualized using tSNE (figure 1A-B) and manual gating. Further, phospho-specific flow cytometry was used to measure basal levels of pERK, pPLCg2 and p38 and expression following CD3/CD28 (TCR) and anti-IgG and IgM (BCR) stimulation. Whole genome RNA-sequencing was performed on flow cytometry sorted T cells, B cells and monocytes from 35 matched ANA-, ANA +and SLE patients followed by weighted correlation network analysis (WGCNA) and pathway enrichment analyses.

Results Both European and African American SLE patients were distinguished from healthy individuals by $\mathrm{T}$ cell proliferation $(p=0.002)$ (figure $1 \mathrm{C}$ ), plasmacytoid dendritic cell activation $(p=0.021)$ and elevated stem cell factor $(p=0.0003)$. EA ANA+healthy individuals exhibited greater immune regulation with reduced $T$ cell numbers $(p=0.002)$ (figure $1 C)$, decreased activation of dendritic cells $(\mathrm{p}=0.039)$ and transitional $\mathrm{B}$ cells (0.033), and elevated expression of the inhibitory receptor CD85j $(p=0.042)$ on specific immune cell subsets compared to ANA- healthy subjects. Further, a module associated with hematopoiesis, $\mathrm{T}$ cell activation and intrinsic apoptosis signaling pathways is expressed at a higher level in $\mathrm{T}$ cells of EA ANA+individuals. In contrast, AA ANA+healthy individuals had elevated plasma levels of IL-6 $(p=0.018)$ and reduced inhibitory receptor expression $(p=0.0089)$ compared to ANAhealthy controls. Gene expression modules associated with viral responses and type I IFN pathway activation were identified in AA SLE patient B cells, while similar expression modules were only found in the monocytes of European American SLE patients.

Conclusions These results highlight the importance of stem cell factor and $\mathrm{T}$ cell expansion in SLE pathogenesis, and suggest that mechanisms of SLE pathogenesis differ by ethnicity. ANA +European Americans may have more effective regulatory mechanisms in place to prevent transition to classified autoimmune disease.

Funding Source(s): This work was supported by the NIH under award numbers U54GM104938,

U01AI101934, U19AI082714, and P30AR053483.

\section{CLINICIANS WITH LUPUS LOSING SKILLS AND DIVERSITY FROM HEALTH SERVICES RESULTS FROM AN ON-LINE SURVEY}

${ }^{1}$ Sara Booth*, ${ }^{2}$ Elizabeth Price, ${ }^{3}$ Elizabeth Walker. ${ }^{1}$ University of Cambridge; ${ }^{2}$ Hull York Medical School; ${ }^{3}$ Faculty of Medical Sciences

10.1136/lupus-2019-|sm.234

Background It is well-documented that people with systemic lupus erythematosus (SLE) have high levels of workplace disability and unemployment. We present the data from a sub-group of participants in an on-line survey designed to understand the barriers and facilitators to employment in people with lupus as a first step towards solutions. Three hundred and ninety-three detailed questionnaires were returned, demonstrating that the overarching barriers to continuing employment were fluctuation, invisibility and fatigue, facilitators were flexibility in working pattern, parttime working understanding management and colleagues. Participants suffered from high levels of anxiety and distress about their loss of income and likely future inability to work. Those claiming benefits felt guilt, shame and humiliation.

Methods The on-line survey was conducted in UK adults with SLE, through the LUPUS UK website, and was designed to find out about the difficulties and successes that people with SLE have in maintaining employment. The data from participants working in a clinical profession or employed by the National Health Service (NHS) in a clinicalrelated activity were identified from the cohort. Their data is currently being analysed to see if those working in NHS organisations have consistently good experiences of workplace support and better chances of retention.

Results Fifty-five of the sample of 393 (14\%) worked or had worked in the NHS in a clinical or clinically-related role. Roles include doctors, managers clinical coders, midwives, podiatrists, pharmacists, operating department assistants. The largest group were nurses including two in senior roles and untrained nursing assistants. Most of the sample had reduced hours or stopped working completely directly as a result of lupus, support was variable with some attributing workplace flexibility to NHS employment but others experiencing harassment. A recently introduced method of calculating sick leave militates against people with fluctuating conditions.

Conclusions SLE presents specific difficulties for maintaining employment fatigue, fluctuation and invisibility not addressed by current anti-discrimination legislation or currently-available reasonable adjustments. As Europes largest employer with a core mission of treating illness the NHS could set an example of how to overcome barriers to employment in people with fluctuating conditions, like lupus. There is clear evidence of loss of skills from the organisation and emerging evidence from this study suggest variable support and levels of knowledge form managers and colleagues. Personal pain from loss of role and income were high in participants. Further data will be available.

Funding Source(s): No external funding required

\section{IKZF1 AND IKZF3 INHIBITION IMPAIRS B CELL DIFFERENTIATION AND MODULATES GENE EXPRESSION IN SYSTEMIC LUPUS ERYTHEMATOSUS}

${ }^{1}$ Sotiria Manou-Stathopoulou*, ${ }^{1}$ Felice Rivellese, ${ }^{1}$ Daniele Mauro, ${ }^{1}$ Katriona Goldmann, ${ }^{2}$ Debasish Pyne, ${ }^{3}$ Peter Schafer, ${ }^{1}$ Michele Bombardieri, ${ }^{1}$ Costantino Pitzalis, ${ }^{1}$ Myles Lewis. ${ }^{1}$ Queen Mary University of London; ${ }^{2}$ Barts Health NHS Trust; ${ }^{3}$ Celgene Corporation

10.1136/lupus-2019-Ism.235

Background Polymorphisms in IKZF1 (Ikaros) and IKZF3 (Aiolos), which are transcriptions factors essential for $\mathrm{B}$ cell activation, maturation and differentiation, have been associated with Systemic Lupus Erythematosus (SLE). However the functional role of IKZF1 and IKZF3 in the context of failed B 